\title{
Extremely brief mindfulness interventions for women undergoing breast biopsies: a randomized controlled trial
}

\author{
Bertha Andrade Coelho ${ }^{1,2}$. Sara de Pinho Cunha Paiva ${ }^{3}$. Agnaldo Lopes da Silva Filho ${ }^{1,3}$
}

Received: 6 May 2018 / Accepted: 26 June 2018 / Published online: 5 July 2018

(c) Springer Science+Business Media, LLC, part of Springer Nature 2018

\begin{abstract}
Purpose Mindfulness-based programs can reduce stress and help practitioners to have positive attitudes in their daily lives. This randomized controlled trial evaluated the impact of brief Mindfulness interventions on quantitative and qualitative stress parameters in patients undergoing imaging-guided breast biopsies.

Methods Eighty-two women undergoing percutaneous imaging-guided breast biopsy were randomized into two groups: MBI group or standard care group. One week before the biopsy procedure, on the waiting room and during the biopsy procedure, the MBI group was exposed to mindfulness techniques and the standard care group received supportive dialogue from the biopsy team. Participants completed questionnaires measuring depression, anxiety and stress, demographics, and medical history, besides evaluating their pain experience through a visual analogue scale for pain and had their systolic and diastolic blood pressure, initial and final temperate, heart rate, oxygen saturation, and salivary cortisol measured.

Results Participation in the mindfulness intervention group was associated with reduced levels of perceived stress, blood pressure, heart rate, and oxygen saturation compared to participation in the standard care group $(P$ values $<0.05)$. No difference was observed regarding salivary cortisol levels, peripheral temperature, and pain perception between the two studied groups.

Conclusion Results indicate that an extremely brief mindfulness intervention is a feasible intervention, suggesting that Mindfulness-based programs may be beneficial to reduce discomfort in acutely stressful settings.
\end{abstract}

Keywords Mindfulness $\cdot$ Breast biopsy $\cdot$ Stress markers $\cdot$ Salivary cortisol

\section{Introduction}

Breast cancer is the most common cancer in women worldwide [1]. Annually, millions of women are screened for early detection of breast cancer, and there is no universally accepted screening strategy so far. It is estimated that among all women who undergo screening mammograms, $10.6 \%$ of

Bertha Andrade Coelho

budcoelho@gmail.com

1 Department of Gynecology and Obstetrics of the School of Medicine of UNESP - Universidade Estadual Paulista "Júlio de Mesquita Filho", Distrito de Rubião Júnior s/no, 18.618-970 Botucatu, São Paulo, Brazil

2 Faculty of Medicine, FIP-MOC, Faculdades Integradas Pitágoras de Montes Claros, Montes Claros, MG, Brazil

3 Department of Gynecology and Obstetrics, Faculty of Medicine, UFMG, Federal University of Minas Gerais, Belo Horizonte, MG, Brazil them will be recalled to additional tests, including breast biopsies [2].

Guided biopsies are outpatient procedures performed by breast radiologists under ultrasound or stereotaxic guidance to diagnose abnormalities identified during breast propaedeutic. Core biopsy (CB) is an outpatient procedure in which cylindrical tissue samples are obtained [3]. It is a procedure that lasts approximately $20 \mathrm{~min}$, is affordable, and analgesic medication prescription is rarely necessary after the intervention [4]. However, it is associated to stress for many women, followed by insomnia, difficulty on concentrating, and high levels of anxiety before and during the procedure [3]. It is also noteworthy that high levels of anxiety can interfere with patients' adherence to screening recommendations [3].

A recent study found that patients demonstrated higher anxiety as waiting time to breast biopsies increased [3]. However, as most patients do not have the procedure performed on the same day of biopsy indication, psychosocial 
interventions, such as Mindfulness relaxation protocols, may reduce anxiety prior and during the biopsy.

Meditation can be defined as a form of mental training that aims to improve an individual's basic psychological capacities, such as attention and emotional control [5-7]. The practice of meditation is the framework used for development of the state or ability in mindfulness. The word "meditation" comes from the Latin word meditari, which means to participate in contemplation [8]. Mindfulness meditation is often described as a non-judgmental technique to experience being in the present moment $[6$, 7, 9-11], and its core components are attention control, emotional regulation, and self-awareness. In 1979, Jon Kabat-Zinn included the Mindfulness-based stress reduction program to the treatment of patients with chronic pain and demonstrated that the way patients dealt with pain can change their own experience of pain $[12,13]$. Mindfulness can be practiced through various forms of meditation. Therefore, interventions such as meditation, breathing exercises, guided imagery, relaxation methods, and Yoga, for example, can be understood as Mindfulnessbased interventions (MBIs) [11].

Traditional Mindfulness training programs are extensive and conducted by experienced specialists. An important barrier to the widespread implementation of Mindfulness programs is the time required for training and practice. To illustrate, the program developed by Jon Kabat-Zinn includes weekly sessions of $2.5 \mathrm{~h}$ of practice and didactic classes for 8 consecutive weeks, a 1-day retreat in silence, and a recommendation for $45 \mathrm{~min}$ of daily practice meditation [1, 10,11]. Although these programs are effective and highly valued, not all patients are interested or have the resources or time available to participate in these long-term trainings [14] and although traditional programs are structured on training over several months $[6,15]$, some studies suggest that targeted interventions on mindfulness training produce measurable positive results on pain, anxiety, fatigue, and sleep for the studied populations $[4,7,11,14,16]$.

Brief interventions can be practiced by individuals in their own settings, with lower costs and minimal training $[11,14]$. In a systematic review of the literature, brief Mindfulness intervention was defined as those that last less than $4 \mathrm{~h}$ [11]. Mindfulness-based programs reduce stress in different settings and help practitioners to have positive attitudes in their daily lives. A previous study demonstrated that guided meditation and music interventions reduce patient anxiety and fatigue [4], signalizing that these interventions during breast biopsies provide greater comfort and tolerability for the patients. Therefore, the key research question of this randomized controlled trial was whether an extremely brief mindfulness intervention prior to breast biopsy could reduce quantitative and qualitative stress parameters on women exposed to this procedure.

\section{Methods}

\section{Study design and ethical approval}

An open and controlled intervention trial was conducted, and potential participants were those attending to an outpatient radiologic clinic to perform a breast biopsy under local anesthesia from August to December 2017. Patients had to be literate and older than 18 years old. Non-including criteria were previous treatment with chemotherapy, previous diagnosis of immune system disorder, regular use of immunosuppressants, corticosteroids and/ or anti-inflammatories in the last 3 months, women who had undergone breast biopsy in the previous 6 months, and non-agreement to participate in the study. To test the differences between means and assuming a large effect size (0.80), significance level of $5 \%$ and a statistical power equal to or greater than 0.80 , a sample size of 41 individuals for each group was settled.

Patients were randomized to the groups every other week of the biopsy schedule, so that all participants on a given day of biopsy were included in the same intervention group. Patients in the intervention group were invited to participate in the study on the week prior to the procedure and the patients in the standard-care control group were invited to participate on the procedure day. All biopsies were performed on Tuesdays after $4 \mathrm{pm}$. Patients' flowchart is represented in Fig. 1. Clinical and sociodemographic data and vital signs were collected prior to the biopsy procedure. The study was approved under the regimens of the local Research Ethics Committee (Faculty of Medicine-FIP-MOC, Minas Gerais, Brazil) and all patients received information on the research objectives, procedures, and data confidentiality and signed the informed consent form.

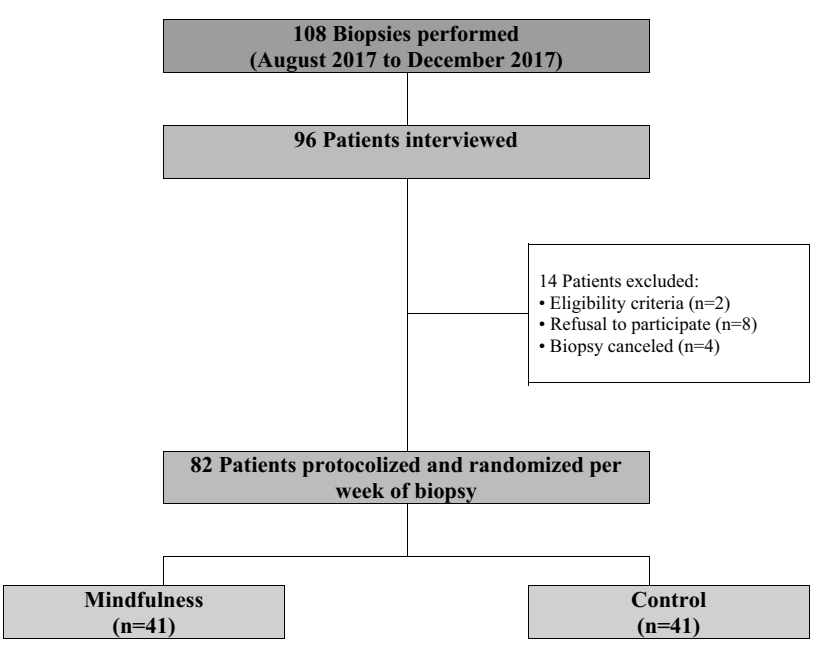

Fig. 1 Included patients flowchart 


\section{Interventions and outcome measures}

Patients were allocated to intervention or standard-care control group on intercalated weeks. For the intervention group, all eligible patients who had a breast biopsy scheduled were telephoned and invited to participate in the study on the week prior to the intervention. Participants were blinded to the purpose of the study and were informed that the aim of the study was to evaluate some stress markers in patients submitted to breast biopsies and not that the main objective was to measure the mindfulness impact on quantitative and qualitative parameters of stress markers, to reduce information bias. Since the consent was given, in consecutive days an audio or video of a Mindfulness-based body scan was sent to the patients through an electronic message. Body scan techniques were chosen due to its simplicity since the patients did not need to have any prior knowledge or training in meditation methods. These techniques involve concentration in the present moment through the observation of breathing and bodily sensations, and the audios and videos encouraged the patients to a state of a non-judgemental acceptance of thoughts and feelings experienced in the moment. Patients included in the control group were those attending to the clinic following the standard-care protocol and received usual orientation from the biopsy team.

On the biopsy day, the patients responded to a socioeconomic questionnaire. Patients on the intervention group waited for the procedure in a quiet and silent room with meditation videos, which associates relaxing music and nature landscapes images, while the standard-care control group remained in the waiting room watching a regular television program. During the biopsy procedure, the Mindfulness patients also heard to a body scan audio (produced by our work group for previous research at Federal University of Minas Gerais, described elsewhere [17]) and were instructed to focus on it while the biopsy was performed. The orientations given during the procedure were the same for the two groups studied, and the audio did not prevent communication between medical staff and patients.

To evaluate stress markers, quantitative (systemic blood pressure, heart rate, peripheral oxygen saturation, initial and final digital temperature, and salivary cortisol) and qualitative parameters (Depression, Anxiety, and Stress Scale and Visual Analogue Scale for Pain) were examined. The 21-item Depression, Anxiety, and Stress Scale (DASS-21) is a set of three assessment scales used to measure emotional states of depression, anxiety, and stress. Each of the three DASS-21 subscales contains 7 items with similar content. The depression scale evaluates dysphoria, hopelessness, self-depreciation, lack of interest/involvement, anhedonia, and inertia. The anxiety scale assesses autonomic arousal, musculoskeletal responses, situational anxiety, and subjective affectivity experience. The stress scale assesses difficulty in relaxing, nervous excitement, irritated, or impatient mood. The Visual Analogue Scale (VAS) of pain is a onedimensional measure of pain intensity, which is widely used in several populations (Fig. 2). All the questionnaires were responded, and the vital signs measured minutes prior to the biopsy. The final temperature was measured, and VAS was responded just after the biopsy and the salivary cortisol samples were collected and identified $30 \mathrm{~min}$ after the procedure and were subsequently centrifuged and stored until the laboratory analyses, which was performed by electrochemiluminescence.

\section{Statistical analyses}

Descriptive statistics were used to summarize data on demographics and clinical variables. Data were analyzed using Statistical Package for the Social Sciences-SPSS (version 190.0 ). Data normality distribution was evaluated by the Kolmogorov-Smirnov test and the statistical differences between groups were tested using Student's t-test. The

Fig. 2 Visual abstract

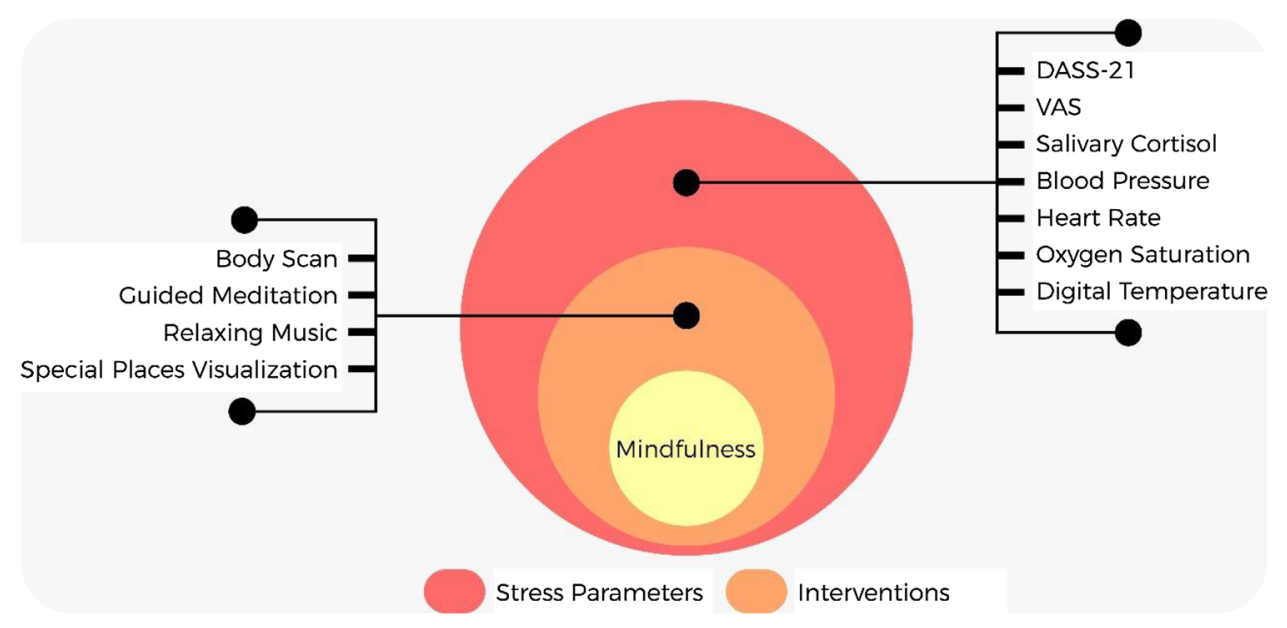


association between groups and qualitative variables were tested using Chi-Squared test. Significance level was set at $5 \%$.

\section{Results}

\section{Descriptive statistics}

Eighty-nine percent (86/96) of eligible patients provided consent for the present study, and 95\% (82/86) of consented patients were evaluable. Eighty-two patients participated in this study, 41 women in the standard-care control group and 41 in the intervention group (Fig. 1). One hundred and eight biopsies were performed during the recruitment period, but 12 patients had their biopsy performed within $48 \mathrm{~h}$ after the medical request and were in the randomized week for the intervention group. Since there was not enough time for these patients to be invited to participate in the study and to be exposed to the mindfulness interventions prior to the breast biopsy, they were not included in the study population. The age of the subjects ranged from 18 to 83 years, with a mean age of 44.3 years. Most of the subjects were married (67.1\%) and only $24.4 \%$ of them had an occupation that required a bachelor's degree or a higher degree. The majority (76.6\%) of the studied population had a family income of 1-6 national minimum wages per month. Most of the patients were not used to meditation practices and had no psychologic counseling (91.5\%). Catholicism was the most prevalent religion, accounting for $57.3 \%$ of the participants. Family history for breast cancer was present in $30.5 \%$ of the women evaluated, and $18.3 \%$ of them had already undergone breast biopsies.

There were no differences between groups regarding age, age at first birth, parity, working and schooling time, marital status, profession, meditation practice, religion, psychological support, previous breast biopsies, or family history of breast cancer. Table 1 presents the summary statistics for clinical and demographic data.

Concerning the quantitative stress parameters, systolic and diastolic blood pressure levels in the intervention group were significantly lower $(P=0.04$ and $P=0.002$,
Table 1 General sample characteristics $(n=82)$

\begin{tabular}{|c|c|c|c|c|}
\hline \multirow[t]{2}{*}{ Variables } & \multirow[t]{2}{*}{ Categories } & \multicolumn{2}{|l|}{ Groups } & \multirow[t]{2}{*}{$P$} \\
\hline & & Mindfulness $n=41$ & Control $n=41$ & \\
\hline Age (y) & & $44.7( \pm 15.9)$ & $43.9( \pm 11.8)$ & $0.786^{\mathrm{a}}$ \\
\hline Age at first birth (y) & & $23.8( \pm 5.4)$ & $23.7( \pm 5.7)$ & $0.934^{\mathrm{a}}$ \\
\hline Parity & & $1.9( \pm 1.6)$ & $2.0( \pm 1.7)$ & $0.690^{\mathrm{a}}$ \\
\hline Working time (y) & & $18.3( \pm 11.7)$ & $15.1( \pm 10.6)$ & $0.233^{\mathrm{a}}$ \\
\hline Schooling time $(\mathrm{y})$ & & $15.1( \pm 5.7)$ & $16.9( \pm 8.1)$ & $0.245^{\mathrm{a}}$ \\
\hline \multirow[t]{4}{*}{ Marital status } & Married & $70.7 \%$ & $63.4 \%$ & $0.735^{\mathrm{b}}$ \\
\hline & Single & $14.6 \%$ & $22 \%$ & \\
\hline & Widow & $4.9 \%$ & $4.9 \%$ & \\
\hline & Divorced & $9.8 \%$ & $9.8 \%$ & \\
\hline \multirow[t]{4}{*}{ Profession } & Housewife & $19.5 \%$ & $31.7 \%$ & $0.351^{\mathrm{b}}$ \\
\hline & $\begin{array}{l}\text { Occupation that requires } \\
\text { a Bachelor's degree or } \\
\text { higher }\end{array}$ & $29.3 \%$ & $19.5 \%$ & \\
\hline & Retired & $9.8 \%$ & $4.3 \%$ & \\
\hline & Other & $41.5 \%$ & $43.9 \%$ & \\
\hline Meditation practice & Yes & $9.8 \%$ & $7.3 \%$ & $0.697^{\mathrm{b}}$ \\
\hline \multirow[t]{4}{*}{ Religion } & Non & $9.8 \%$ & $4.9 \%$ & $0.865^{\mathrm{b}}$ \\
\hline & Catholic & $53.9 \%$ & $61 \%$ & \\
\hline & Protestant & $34.1 \%$ & $31.7 \%$ & \\
\hline & Spiritualist & $2.4 \%$ & $2.4 \%$ & \\
\hline Psychological support & Yes & $9.8 \%$ & $7.3 \%$ & $0.697^{\mathrm{b}}$ \\
\hline Previous breast biopsy & Yes & $19.5 \%$ & $17.1 \%$ & $0.778^{\mathrm{b}}$ \\
\hline Family history of breast cancer & Yes & $29.3 \%$ & $31.7 \%$ & $0.813^{\mathrm{b}}$ \\
\hline
\end{tabular}


respectively) when compared to the standard-care control group as well as their heart rate $(P=0.016)$ and peripheral oxygen saturation level $(P=0.026)$ (Table 2$)$. No significant differences between groups were found when initial and final temperature and salivary cortisol levels were examined.

The analyses of the qualitative stress parameters showed that there was a significant difference between the two groups when looking at the DASS-21 stress subscale, where the Mindfulness patients presented lower scores $(P=0.029)$ (Table 3). There were no group differences in the depression and anxiety subscales and pain rating after the biopsy.

Additionally, $87 \%$ of the patients questioned about the utility of the Mindfulness intervention considered the strategy very or extremely helpful, and $87 \%$ of them would strongly recommend those same interventions to other patients in preparation for interventional procedures (Fig. 3).

\section{Discussion}

Significant levels of stress and anxiety are reported among women who are in preparation for breast biopsies [18] and a better understanding of the factors that contribute to this discomfort and the development of strategies to help patients to manage stress are needed [3]. This study examined the effects of an extremely brief mindfulness intervention for women undergoing breast biopsies. Traditionally, studies that evaluate Mindfulness-based interventions deal with chronic diseases scenarios, such as cancer, arthritis, diabetes, psychiatric disorders [19]. Instead, our study evaluated the repercussion of a punctual mindful intervention in an acute stress scenario in otherwise healthy women.

Our results indicated that women in the mindfulness group presented lower levels of stress compared to women in the standard care group. This finding indicates that the Mindfulness-based intervention-which guided participants to bring their awareness to the present moment and observe their experience non-judgmentally with curiosity and a sense of allowing - is an effective method for reducing stress during a medical procedure, which is commonly associated with high distress [5]. Previous researches which also used the DASS-21 to measure the outcomes of MBIs have also supported that mindfulness interventions can decrease depression, anxiety, and stress for clinical and non-clinical populations, regardless of the hours of exposure to the interventions [20-23]. It is also noteworthy that the use of
Table 2 Group statisticsquantitative parameters

\begin{tabular}{|c|c|c|c|c|c|}
\hline \multirow[t]{3}{*}{ Variables } & \multicolumn{4}{|l|}{ Groups } & \multirow[t]{3}{*}{$P$} \\
\hline & \multicolumn{2}{|l|}{ Mindfulness } & \multicolumn{2}{|l|}{ Control } & \\
\hline & Mean $( \pm S D)$ & [Min; Max] & Mean $( \pm S D)$ & [Min; Max] & \\
\hline SP & $122.9( \pm 20.8)$ & {$[90 ; 160]$} & $137.3( \pm 22.8)$ & {$[100 ; 190]$} & 0.004 \\
\hline DP & $75.6( \pm 11.8)$ & {$[60 ; 100]$} & $86( \pm 16.5)$ & {$[60 ; 130]$} & 0.002 \\
\hline Initial temperature & $28.3( \pm 2.6)$ & {$[22.3 ; 33.6]$} & $28.3( \pm 2.3)$ & {$[25 ; 33]$} & 0.898 \\
\hline Final temperature & $26.9( \pm 2.9)$ & {$[21.1 ; 33.3]$} & $27.7( \pm 2.9)$ & {$[23.9 ; 33.7]$} & 0.261 \\
\hline HR & $75.5( \pm 10.8)$ & {$[59 ; 97]$} & $81.7( \pm 11.4)$ & {$[60 ; 105]$} & 0.016 \\
\hline Oxygen saturation & $95.1( \pm 3.8)$ & {$[79 ; 99]$} & $96.7( \pm 1.9)$ & {$[92 ; 99]$} & 0.026 \\
\hline Salivary cortisol & $5.4( \pm 4.6)$ & {$[1.5 ; 22]$} & $4.3( \pm 3.6)$ & {$[1.4 ; 16.9]$} & 0.258 \\
\hline
\end{tabular}

Comparisons between groups by the Student's $t$ test

Data expressed as means \pm standard deviations

$S P$ systolic pressure, $D P$ diastolic pressure, $H R$ heart rate

\begin{tabular}{lllllll}
\hline Variables & \multicolumn{3}{l}{ Groups } & & $P$ \\
\cline { 2 - 3 } & \multicolumn{1}{l}{ Mindfulness } & & \multicolumn{2}{l}{ Control } & \\
\cline { 2 - 3 } & Mean $( \pm$ SD $)$ & {$[$ Min; Max $]$} & & Mean $( \pm$ SD $)$ & {$[$ Min; Max $]$} & \\
\hline DASS stress & $12.8( \pm 9.6)$ & {$[0 ; 34]$} & & $18.1( \pm 12)$ & {$[0 ; 42]$} & 0.029 \\
DASS anxiety & $6.9( \pm 6.9)$ & {$[0 ; 30]$} & & $9.4( \pm 8.2)$ & {$[0 ; 32]$} & 0.133 \\
DASS depression & $7.8( \pm 8.4)$ & {$[0 ; 34]$} & & $10.6( \pm 11.5)$ & {$[0 ; 42]$} & 0.209 \\
VAS & $1.3( \pm 1.1)$ & {$[0 ; 4]$} & & $1.5( \pm 1.2)$ & {$[0 ; 6]$} & 0.634 \\
\hline
\end{tabular}

Data expressed as means \pm standard deviations. Comparisons between groups by the Student's $t$ test DASS Depression Anxiety Stress Scale Short Form 21, VAS Visual Analogue Scale 


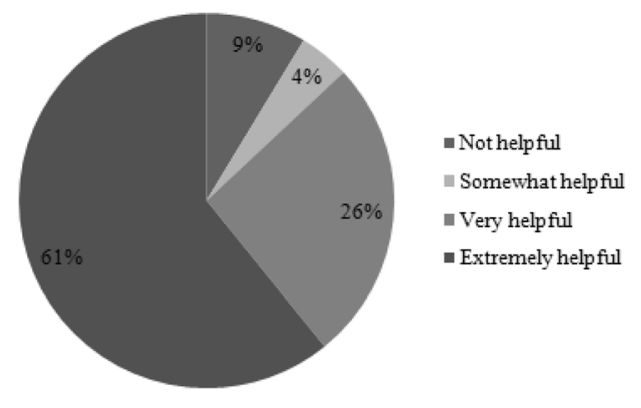

A - How helpful was listening to the audio guide to help you relax?

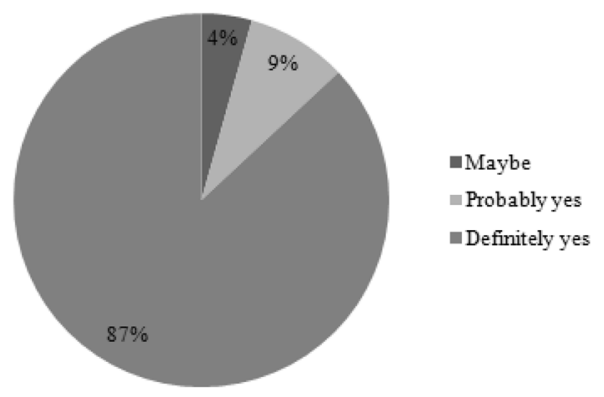

B - You would recommend this strategy to others who are trying to manage anxiety before biopsies?

Fig. 3 Patients' perception on utility and mindfulness intervention recommendation to other women undergoing breast biopsies

audio-recorded-guided meditation is simple and inexpensive to implement in outpatient settings, with fewer drawbacks compared with other anxiety reducing techniques [4] and our findings support the effectiveness of a simple and brief MBI as a stress management strategy.

Concerning the vital signs, our results demonstrated that participation in the MBI group is associated with reduced levels of systolic and diastolic blood pressure, heart rate, and oxygen saturation compared to participation in the standard care group. Those findings were also described in previous studies $[21,24]$, regardless the subjects were exposed to acute or chronic mindful interventions or practices. The vital signs profile changes are probably related to decreased arousal and a decrease in sympathetic nervous system activity.

Our study was not able to demonstrate a significant reduction in cortisol secretion after a brief mindfulness intervention. This finding agreed to a recent metanalyses result, which concluded that MBIs showed moderately low effects in improving the state of health related to cortisol levels in healthy individuals [25]. It also showed that there was a tendency to more pronounced effects under conditions where interventions were conducted with a higher number of sessions and hours of mindfulness training - the studies that conducted MBIs under conditions with more than $20 \mathrm{~h}$ of programmed training seemed to have more effective impacts on salivary cortisol levels [25].

Contrary to our hypotheses, there was no significant group difference for pain perception in this study. The Visual Analogue Scale for Pain (VAS Pain) is widely used due to its simplicity and adaptability to a broad range of populations and settings $[26,27]$ and some researchers have indicated that short mindfulness training can result in immediate reduction in acute pain intensity [28]. But interestingly, our results show that using a $10-\mathrm{cm}$ length VAS, the average scores for the mindfulness and standard groups were 1.3 and
$1.4 \mathrm{~cm}$, respectively, which agreed with previous research [4], suggesting that pain itself is not a major issue during breast biopsies. To corroborate this impression, a study that evaluated pain experience during image-guided breast biopsy procedures, revealed that from 298 patients who underwent ultrasound-guided biopsy, $71 \%$ of them reported no or mild pain during the procedure [29].

In summary, our study demonstrated that participation in the mindfulness intervention group was associated with reduced levels of perceived stress, blood pressure, heart rate, and oxygen saturation compared to participation in the standard care group. No difference was observed regarding salivary cortisol levels, peripheral temperature, and pain perception between the two studied groups.

This study had some limitations and they must be considered. First, our sample size was relatively small, which could introduce bias. In addition, although our study showed significantly lower biopsy stress with MBIs, the sample size of 82 patients may have had underestimated the possible benefit of the intervention itself. A larger, multicenter study could further explore these effects. Second, we did not control the hours each subject was exposed to the MBIs, this can weaken ours and future data analyses. Third, measures of baseline stress parameters pre MBIs were not performed, thus it is unclear how mindfulness training changed stress reactivity from pre to post-exposure. Fourth, the study staff was not blinded to study condition, opening the possibility of behaving differently depending on each group. Finally, the use of an audio-guided mindfulness intervention is both a strength and a limitation of this study. While this approach is replicable in different settings, the absence of a trained mindfulness instructor for guidance may diminish the efficacy of the training.

Despite these limitations, this randomized controlled trial provides further evidence on the relevance and applicability of MBIs for patients undergoing interventional procedures. 
The findings of this research provide insights for the use of mindfulness intervention in a myriad of settings.

Our results demonstrate that an extremely brief mindfulness intervention is feasible, and strategies based on guided attention may be also beneficial in acute stress scenarios. These results corroborate the findings of previous work addressing the effectiveness of brief mindfulness interventions in reducing stress in different populations. The interventions we proposed are extremely simple, do not require prior training, have minimal costs, and can be implemented in several clinical settings. However, further research is needed to test the generalizability of these findings to more diverse populations.

\section{Compliance with ethical standards}

Conflict of interest The authors declare no conflicts of interest.

Ethical approval All procedures performed in this study were in accordance with the ethical standards of the institutional and national research committee and with the 1964 Helsinki Declaration and its later amendments or comparable ethical standards.

Informed consent Informed consent was obtained from all participants included in the study.

\section{References}

1. Haller H, Winkler MM, Klose P, Dobos G, Kümmel S, Cramer H (2017) Mindfulness-based interventions for women with breast cancer: an updated systematic review and meta-analysis. Acta Oncologica 56:1-12

2. Sickles EA, D’Orsi CJ (2013) ACR BI-RADS® Follow-up and outcome monitoring. In: ACR BI-RADS $®$ Atlas, Breast Imaging Reporting and Data System, 5th edn. American College of Radiology, Reston

3. Hayes Balmadrid MA, Shelby RA, Wren AA, Miller LS, Yoon SC, Baker JA et al (2017) Anxiety prior to breast biopsy: relationships with length of time from breast biopsy recommendation to biopsy procedure and psychosocial factors. J Health Psychol 22(5):561-571

4. Soo MS, Jarosz JA, Wren AA, Soo AE, Mowery YM, Johnson KS et al (2016) Imaging-guided core-needle breast biopsy: impact of meditation and music interventions on patient anxiety, pain, and fatigue. J Am Coll Radiol 13(5):526-534

5. Carlson LE, Garland SN (2005) Impact of mindfulness-based stress reduction (MBSR) on sleep, mood, stress and fatigue symptoms in cancer outpatients. Int J Behav Med 12(4):278-285

6. Rosenzweig S, Greeson JM, Reibel DK, Green JS, Jasser SA, Beasley D (2010) Mindfulness-based stress reduction for chronic pain conditions: variation in treatment outcomes and role of home meditation practice. J Psychosom Res 68(1):29-36

7. Tang YY, Hölzel BK, Posner MI (2015) The neuroscience of mindfulness meditation. Nat Rev Neurosci 16(4):213-225

8. Marchand WR (2014) Neural mechanisms of mindfulness and meditation: evidence from neuroimaging studies. World J Radiol 6(7):471-479

9. Witek-Janusek L, Albuquerque K, Chroniak KR, Chroniak C, Durazo-Arvizu R, Mathews HL (2008) Effect of mindfulness based stress reduction on immune function, quality of life and coping in women newly diagnosed with early stage breast cancer. Brain Behav Immun 22(6):969-981

10. O’Leary K, O’Neill S, Dockray S (2016) A systematic review of the effects of mindfulness interventions on cortisol. J Health Psychol 21(9):2108-2121

11. Gilmartin H, Goyal A, Hamati MC, Mann J, Saint S, Chopra V (2017) Brief mindfulness practices for healthcare providers-a systematic literature review. Am J Med 130(10):1219-1217

12. Matousek RH, Pruessner JC, Dobkin PL (2011) Changes in the cortisol awakening response (CAR) following participation in mindfulness-based stress reduction in women who completed treatment for breast cancer. Complement Ther Clin Pract 17(2):65-70

13. Gotink RA, Chu P, Busschbach JJ, Benson H, Fricchione GL, Hunink MM (2015) Standardized mindfulness-based interventions in healthcare: an overview of systematic reviews and metaanalyses of RCTs. PloS ONE 10(4):1-17

14. Ussher M, Spatz A, Copland C, Nicolaou A, Cargill A, AminiTabrizi $\mathrm{N}$ et al (2014) Immediate effects of a brief mindfulnessbased body scan on patients with chronic pain. J Behav Med 37(1):127-134

15. Cramer H, Lauche R, Paul A, Dobos G (2012) Mindfulnessbased stress reduction for breast cancer-a systematic review and meta-analysis. Curr Oncol 19(5):343-352

16. Nakamura Y, Lipschitz DL, Kuhn R, Kinney AY, Donaldson GW (2013) Investigating efficacy of two brief mind-body intervention programs for managing sleep disturbance in cancer survivors: a pilot randomized controlled trial. J Cancer Surviv 7(2):165-182

17. Ferreira APM, Paiva SPC, Lima SSS, Felicíssimo MF, NogueiraRodrigues A, Carneiro MM et al (2017) The Impact of Mindfulness-based Interventions on the Wellbeing of cervical cancer patients on chemoradiotherapy. Obstet Gynecol Int J 6(6):00091

18. Bugbee ME, Wellisch DK, Arnott IM, Maxwell JR, Kirsch DL, Sayre JW et al (2005) Breast core-needle biopsy: clinical trial of relaxation technique versus medication versus no intervention for anxiety reduction. Radiology 234(1):73-78

19. Zainal NZ, Booth S, Huppert FA (2013) The efficacy of mindfulness-based stress reduction on mental health of breast cancer patients: a meta-analysis. Psycho-Oncology 22(7):1457-1465

20. Gold E, Smith A, Hopper I, Herne D, Tansey G, Hulland C (2010) Mindfulness-based stress reduction (MBSR) for primary school teachers. J Child Fam Stud 19(2):184-189

21. Srinivasan J, Breheny PJ (2012) Meditation for quality improvement of medical encounters: single-intervention, vedanta-based meditation effects on vital signs and mood indices. J Evid Based Complement Alt Med 17(2):96-103

22. Khoury B, Sharma M, Rush SE, Fournier C (2015) Mindfulnessbased stress reduction for healthy individuals: a meta-analysis. J Psychosom Res 78(6):519-528

23. Song Y, Lindquist R (2015) Effects of mindfulness-based stress reduction on depression, anxiety, stress and mindfulness in Korean nursing students. Nurse Educ Today 35(1):86-90

24. Chaya MS, Kurpad AV, Nagendra HR, Nagarathna R (2006) The effect of long term combined yoga practice on the basal metabolic rate of healthy adults. BMC Complement Alt Med 6(1):1-6

25. Sanada K, Montero-Marin J, Díez MA, Salas-Valero M, Pérez-Yus MC, Morillo H et al (2016) Effects of Mindfulness-based interventions on salivary cortisol in healthy adults: a meta-analytical review. Front Physiol 7:1-12

26. Hawker GA, Mian S, Kendzerska T, French M (2011) Measures of adult pain: visual analog scale for pain (VAS Pain), numeric rating scale for pain (NRS Pain), Mcgill pain questionnaire (MPQ), short-form mcgill pain questionnaire (SF-MPQ), chronic pain grade scale (CPGS), short form-36 bodily pain scale (SF-36 
BPS), and measure of intermittent and constant osteoarthritis pain (ICOAP). Arthritis Care Res 63(S11):S240-S252

27. Bird ML, Callisaya ML, Cannell J, Gibbons T, Smith ST, Ahuja KD (2016 Jan) Accuracy, validity, and reliability of an electronic visual analog scale for pain on a touch screen tablet in healthy older adults: a clinical trial. Interact J Med Res 5(1):1-8

28. Garland EL, Baker AK, Larsen P, Riquino MR, Priddy SE, Thomas E et al (2017) Randomized controlled trial of brief mindfulness training and hypnotic suggestion for acute pain relief in the hospital setting. J Gen Intern Med 32(10):1106-1113

29. Pang E, Crystal P, Kulkarni S, Murphy K, Menezes RJ (2016) An audit of pain experienced during image-guided breast biopsy procedures at an academic center. Can Assoc Radiol J 67(3):250-253 